\title{
Development of a flat-field XUV spectrograph for laser plasma interaction studies
}

\author{
A CHOWDHURY, R A JOSHI, S R KUMBHARE, P A NAIK and \\ P D GUPTA
}

Centre for Advanced Technology, Indore 452013, India

e-mail: avijit@cat.ernet.in

MS received 9 December 1998

\begin{abstract}
A flat-field grating XUV spectrograph has been set up for laser plasma interaction studies. It uses a variable groove-spacing concave grating in grazing incidence geometry and operates in the wavelength range of $25-290 \AA$. A brief description of the design principle, construction and operation is given. Variation of spectral resolution with respect to the position of detector plane is studied. A resolution of $0.2 \AA$ (at $\lambda=35.0 \AA$ ) observed from the emission spectrum of carbon plasma is in agreement with that expected from design considerations. The instrument facilitates recording of multiple spectra on a single film and can be coupled to a microchannel plate-CCD camera detection system for on-line monitoring and processing of the spectrum, and to an X-ray streak camera for temporally resolved spectral measurements.
\end{abstract}

Keywords. XUV spectroscopy; flat-field spectrograph; Nd:phosphate glass laser; laser-produced plasmas.

\section{Introduction}

Intense XUV soft X-ray radiation from laser-produced plasma sources is of great current interest for a variety of research investigations like laser-driven inertial confinement fusion (Hogan 1995), XUV-soft X-ray lasing (Mathews et al 1985; Nagata et al 1993; Ditmire et al 1995), very high order harmonic generation (Huillier \& Balcou 1993; Miyazaki \& Takada 1995), and technological applications such as soft X-ray lithography (Chaker et al 1992), X-ray contact microscopy (Stead et al 1995; Chakera et al 1998) etc. Measurements of XUV radiation spectrum of laser-produced plasmas with high temporal and spectral resolutions (Eidmann et al 1986; Chaker et al 1989; Sailaja et al 1998) have become an essential part of laser-plasma interaction experiments. Spectrographs in the XUV soft Xray spectral region commonly use reflection gratings in grazing incidence where the diffracted radiation focuses along the Rowland circle. However, this does not permit coupling of planar detectors like microchannel plate (MCP) and streak cameras to such spectrographs. Use of these detector systems is desirable to facilitate on-line monitoring 
and analysis of the recorded spectrum, and to perform spectral measurements with high temporal resolution ( $\approx$ few picoseconds) respectively.

Reflection gratings have been designed with varied groove-spacing (Kita et al 1983; Nakano et al 1984) to overcome the above difficulty. Radiation diffracted from these gratings is sharply focussed in a plane (flat-field focussing) instead of a curved surface. XUV spectrographs with flat-field focussing have been reported (Kiehn et al 1987; Schwanda et al 1993), using commercially available (Hitachi) variable groove-space gratings. Spectral resolution in these spectrographs is governed by the source size and the linear dispersion, and can be decreased by appropriately reducing the slit width (virtual source). Grazing incidence spherical mirrors are therefore often used to increase the radiation intensity at the slit. However, this complicates the design of the spectrograph and makes it quite bulky and cumbersome in alignment and operation. In principle, a slit of appropriate width can be suitably placed at a small distance from the source to collect enough radiation without using a grazing incidence mirror for a single shot recording of the spectrum with moderate spectral resolution. For instance, in most of the Xray lasing experiments, a spectral resolution of $\leq 0.5 \AA$ is adequate to distinguish the amplified radiation at a desired spectral line from any surrounding structure and in resolving adjacent high odd harmonics in the XUV spectral region of 50-300 (Matthews et al 1985; Huillier \& Balcou 1993; Miyazaki \& Takada 1993; Nagata et al 1993; Ditmire et al 1995).

In this paper, we report on design, construction, and operation of a simple flat-field grating spectrograph using a variable groove-spacing grating for 25-290 $\AA$ spectral range. The spectrograph has a simple mounting arrangement and alignment procedure for the slit and the grating, and facilitates use of various planar detectors, viz. XUV-soft X-ray sensitive film/plates, MCP-CCD camera detection systems, and streak cameras. Variation of spectral resolution with respect to the position of the detector plane is investigated using UFSh-4 films. A resolution of $0.2 \AA$ (at $35.0 \AA$ ) observed from measurements of XUV emission from a laser-produced carbon plasma is in agreement with that expected from design parameters. This simple spectrograph should be suitable for carrying out laser-plasma interaction studies on population inversion and odd harmonic generation in the XUV spectral region.

\section{Description of the spectrograph}

The spectrograph is based on using a variable groove-spacing grating in grazing incidence geometry to achieve flat-field focussing (Kita et al 1983; Nakano et al 1984). A geometrical sketch of the input slit, grating and detector plane for the same is shown in figure 1. Dispersion from the grating is given by the well-known expression, $\sigma(\sin \alpha-\sin \beta)=m \lambda$, where $\sigma$ is the groove spacing, $\alpha$ and $\beta$ are the angles of incidence and diffraction respectively, $m$ the order of diffraction, and $\lambda$ the wavelength of radiation. The grating is a Hitachi make (part no. 001-0266) gold-coated, concave reflection grating with radius of curvature of $5.6 \mathrm{~m}$ and a nominal groove spacing $\sigma$ of $0.83 \mu \mathrm{m}$ corresponding to $1200 \mathrm{~g} / \mathrm{mm}$. It is blazed at $3.2^{\circ}$ for $100 \AA$. The input slit is kept at a distance of $237 \mathrm{~mm}$ from the grating centre at an angle of $87^{\circ}$ with respect to the grating normal. For these conditions, focussing occurs in a plane located at a distance of $235 \mathrm{~mm}$ from the grating centre. Wavelength is then determined as a function of distance $L$ in the focal plane from the foot of the perpendicular to the grating surface at a distance of $235 \mathrm{~mm}$ from the grating centre, as shown in figure 1 . The 


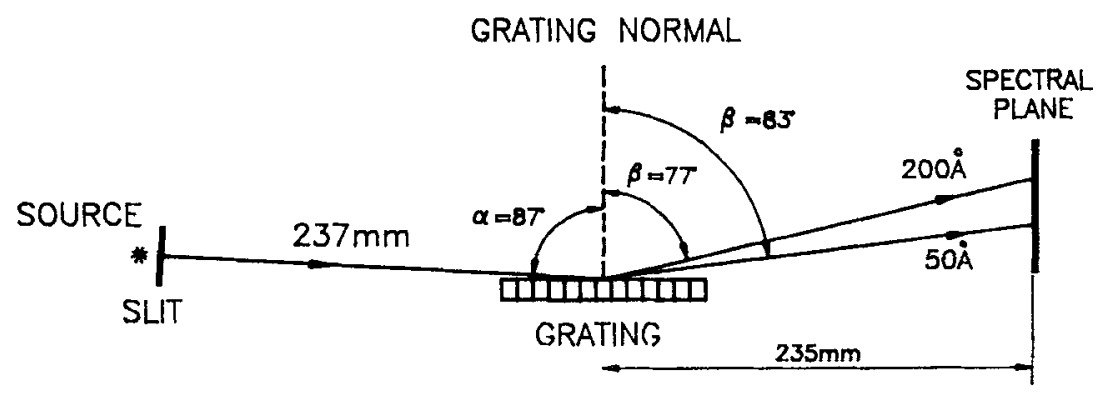

Figure 1. A schematic of the geometry of flat-field focusing.

distance $L(\mathrm{~mm})$ is given by $L=235 \cot \beta$. The plate factor in the focal plane is

$$
\mathrm{d} \lambda / \mathrm{d} L=(\sigma / 235)(\sin \alpha-\lambda / \sigma)^{2}\left[1-(\sin \alpha-\lambda / \sigma)^{2}\right]^{1 / 2} .
$$

It varies from $3.5 \AA / \mathrm{mm}$ at $25 \AA$ to $8.7 \AA / \mathrm{mm}$ at $250 \AA$.

The spectrograph consists of a slit, a light pipe, a grating mount and its housing, a gate valve, a film chamber and a film movement assembly. The top view of the spectrograph assembly is shown in figure 2 , and details of film multi-exposure system are shown in figure 3. A brief description of the spectrograph is as follows. The slit (air slit of width $50 \mu \mathrm{m}$ and height $3 \mathrm{~mm}$ ) is placed vertically at one end of the light pipe that prevents any stray radiation and plasma particles from reaching the grating. The grating is placed in a holder attached to a mount with three micrometer screws. Using these micrometer screws, the grating can be given precise $\left(0.04^{\circ}\right)$ vertical and horizontal tilts as well as movements towards or away from the spectrograph axis. A metal screen whose plane is perpendicular to the grating surface is attached to the grating holder to prevent any direct light from reaching the film. A small aperture ( $1 \mathrm{~mm}$ diameter) is provided in this screen to allow an He-Ne laser beam for purpose of alignment. The mounted grating is enclosed in a light-tight housing. The grating housing is fixed onto a plate mounted on the plasma chamber and is coupled to a light-tight film chamber. A gate valve is used to isolate the plasma chamber from the film chamber whenever the latter is to be opened for loading/unloading the film.

For achieving the best resolution it is necessary to coincide the film plane with the focusing plane of the grating. It is also desirable to be able to record several spectra on different parts of the film along the width to minimize opening of the evacuated film chamber and for better utilization of the film. Both these requirements are accomplished by using the film movement assembly shown in figure 3 . The film is kept in a light-tight cassette mounted on a vertical stepper motor-controlled translational stage ( $\mathrm{Tr}$. stage 1 in figure 3). A mask with an aperture $(55 \mathrm{~mm} \times 3 \mathrm{~mm})$ is placed in front of the film holder. When the film holder is moved down from its initial top position one can get as many as seven non-overlapping spectra on the film. The vertical film movement assembly is mounted on a horizontal translational stage ( $\mathrm{Tr}$. stage 2) operated through a Wilson seal arrangement to coincide the film plane with the focusing plane of the grating.

\section{Experimental}

Experiments were performed using the $2 \mathrm{GW}, 4 \mathrm{~ns} \mathrm{Nd}$ : phosphate glass laser system in the Plasma Radiation Laboratory at the Centre for Advanced Technology. A schematic of the 


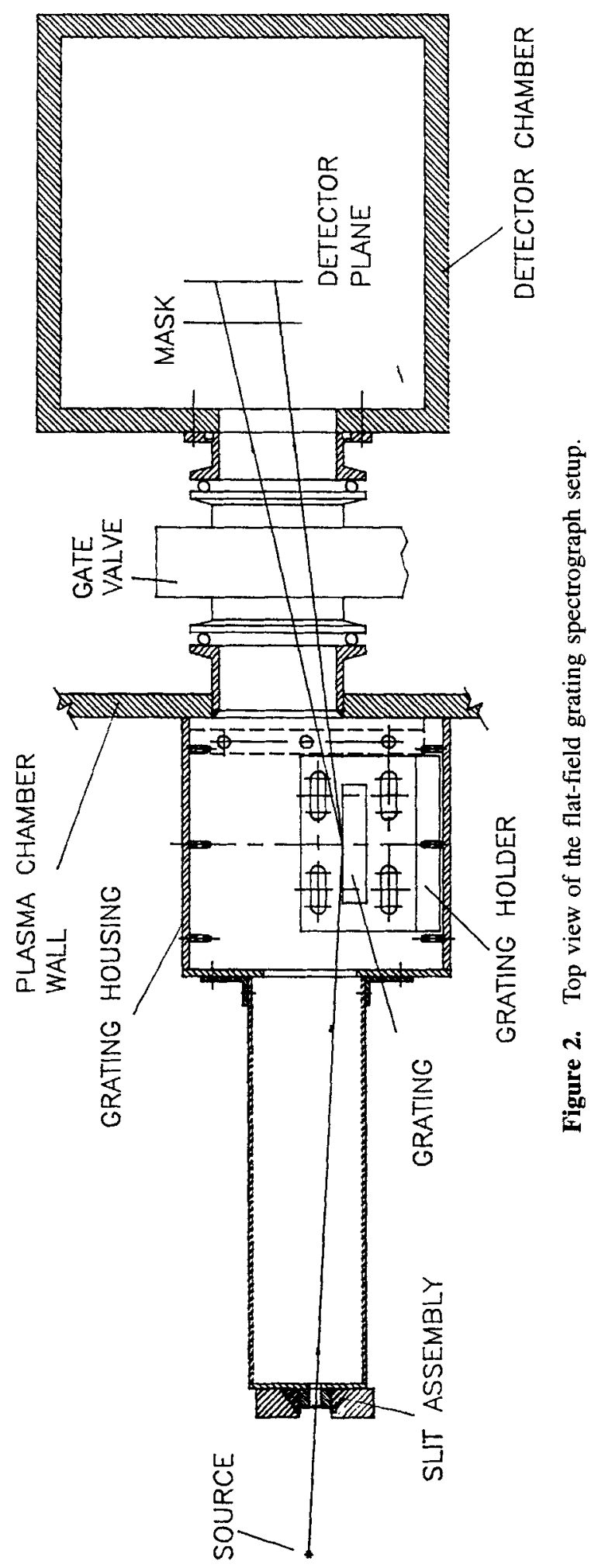




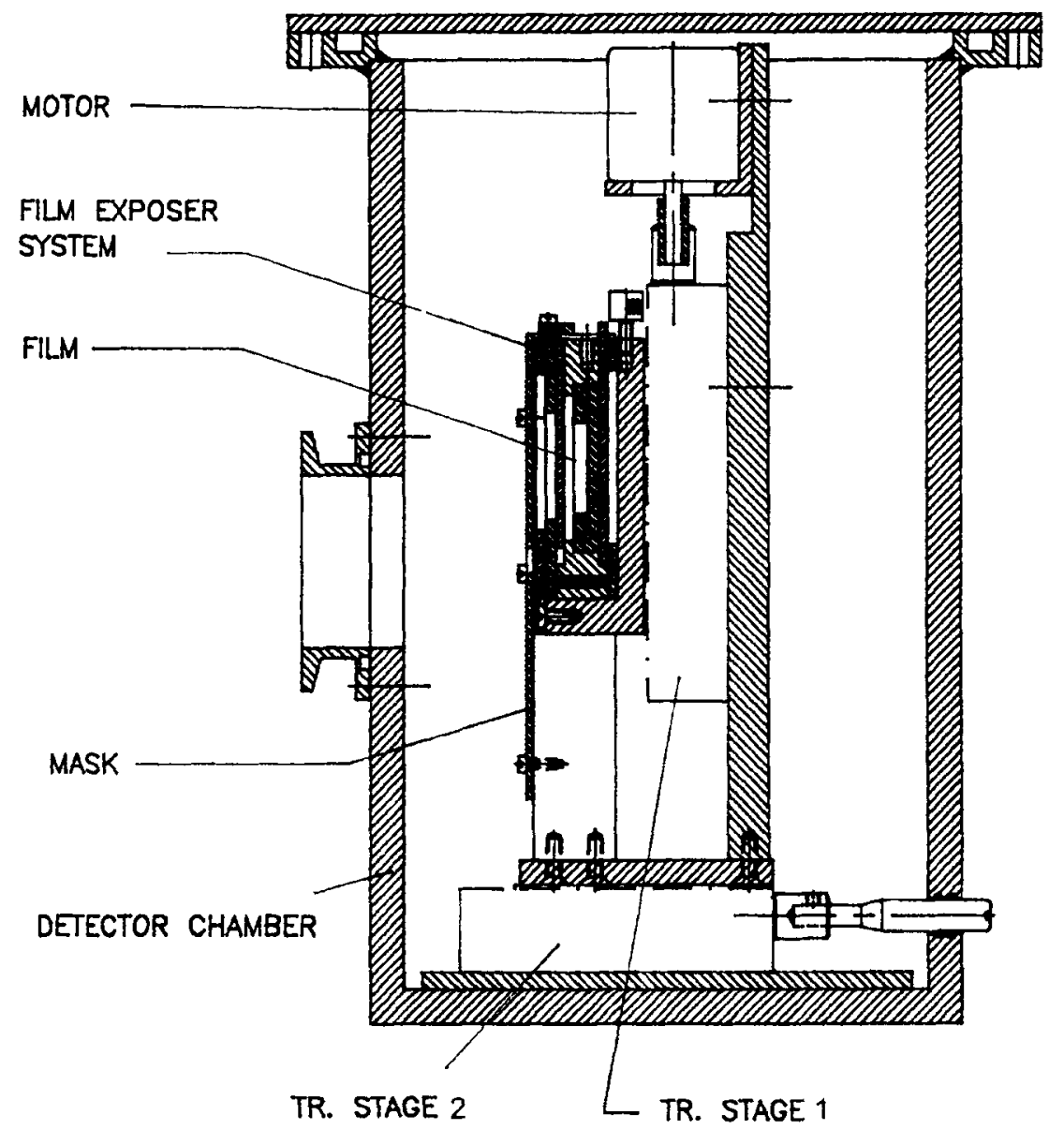

Figure 3. Details of the film multiple-exposure system.

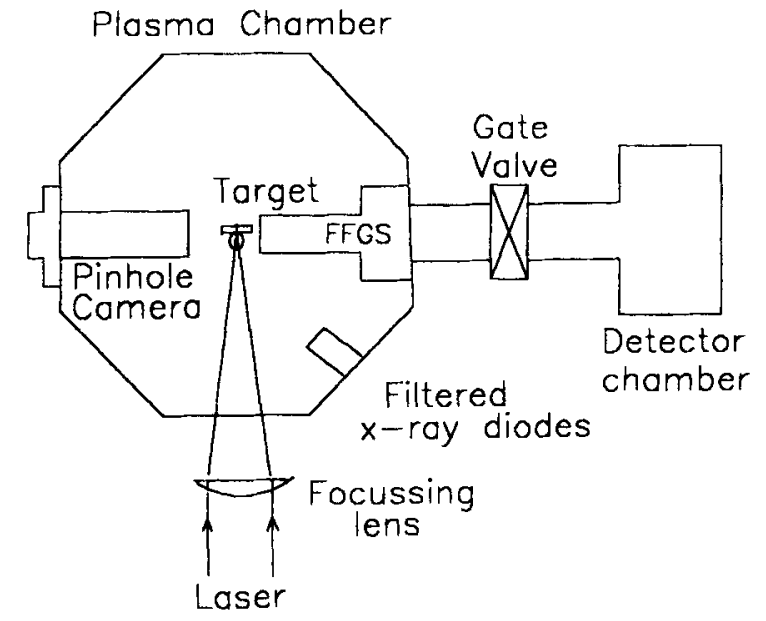

Figure 4. Experimental setup for spectral measurements of laser-produced plasmas. 
experimental setup is shown in figure 4. The laser beam was focussed on planar targets of $\mathrm{Be}, \mathrm{C}, \mathrm{Mg}$ etc. using a 400-millimetre focal length plano-convex lens. Laser shots of energy up to $5 \mathrm{~J}$, were fired on the target. The spectrograph was mounted in an octagonal chamber equipped with demountable flanges and evacuated to $10^{-5}$ torr $(1$ torr $=133 \mathrm{~Pa})$. The spectra were recorded on UFSh-4 Russian films and were developed in a D-19 developer for $6 \mathrm{~min}$ and fixed with a standard acid fixer. Fresh target surface was used for each shot and all the spectra were recorded in a single shot exposure. Typical plasma source size as determined from an X-ray image (filtered through an aluminized $1 \mu \mathrm{m}$ polycarbonate foil $\mathrm{B}-10$ ) recorded using a pinhole camera was $\approx 130 \mu \mathrm{m}$, resulting in an effective laser intensity on the target of $\approx 8 \times 10^{12} \mathrm{~W} / \mathrm{cm}^{2}$. Silicon PIN diodes (Quantrad 100 PIN-250) were also used for measuring integrated X-ray intensities filtered through B-10 foils.

\section{Results and discussion}

XUV emission spectrum of carbon plasma recorded using this spectrograph is shown in figure 5a. An expanded view of the spectrum for $\lambda<44 \AA$ is shown in figure 5b. A $0.8 \mu \mathrm{m}$ thick Formvar $\left(\mathrm{C}_{5} \mathrm{H}_{7} \mathrm{O}_{2}\right)$ filter is kept in front of the film. Due to its carbon constituent, this filter offers strong attenuation to radiation of wavelength just below the carbon $K$-edge $(\lambda=43.7 \AA)$. This results in sharp reduction in transmitted radiation intensity for $\lambda<43.7 \AA$, and the same is clearly visible in the recorded spectrum. This discontinuity serves as a wavelength marker. A densitometric trace of the spectrum of figure $5 \mathrm{~b}$ is shown in figure $5 \mathrm{c}$. The spectral lines are identified using standard data tables (Kelly \& Palumbo 1973). The peaks belong to $\mathrm{He}$-like and $\mathrm{H}$-like emissions of carbon ions. For He-like carbon ions, the transitions are identified to be $\mathrm{CV} 1 s^{2}{ }^{1} S_{0}-1 s 2 p^{1} P_{1}(\mathrm{He} \alpha)$ at $40.3 \AA$, CV $1 s^{2}{ }^{1} S_{0}-1 s 3 p^{1} P_{1}$

(a)
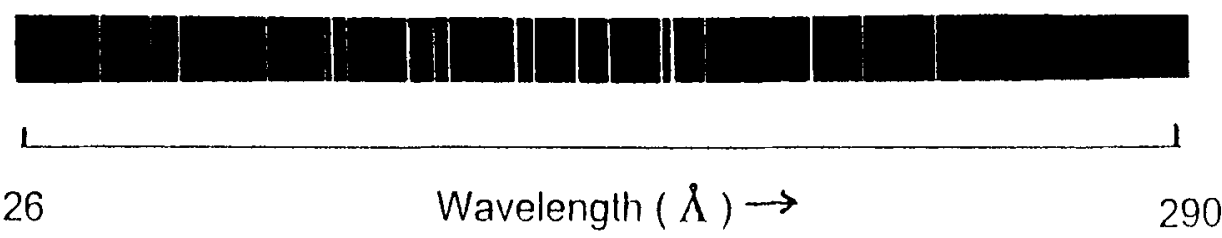

(b)
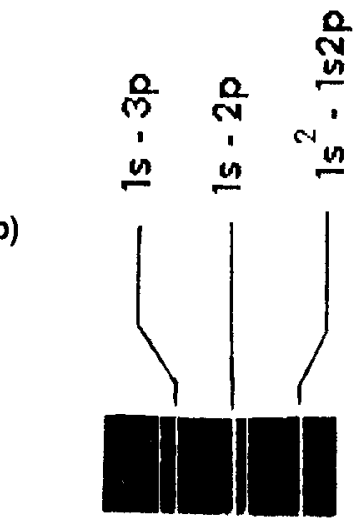

Wavelength $(\AA) \rightarrow$ Figure $5(\mathbf{a}-\mathbf{b}) . \quad$ (Continued.) 


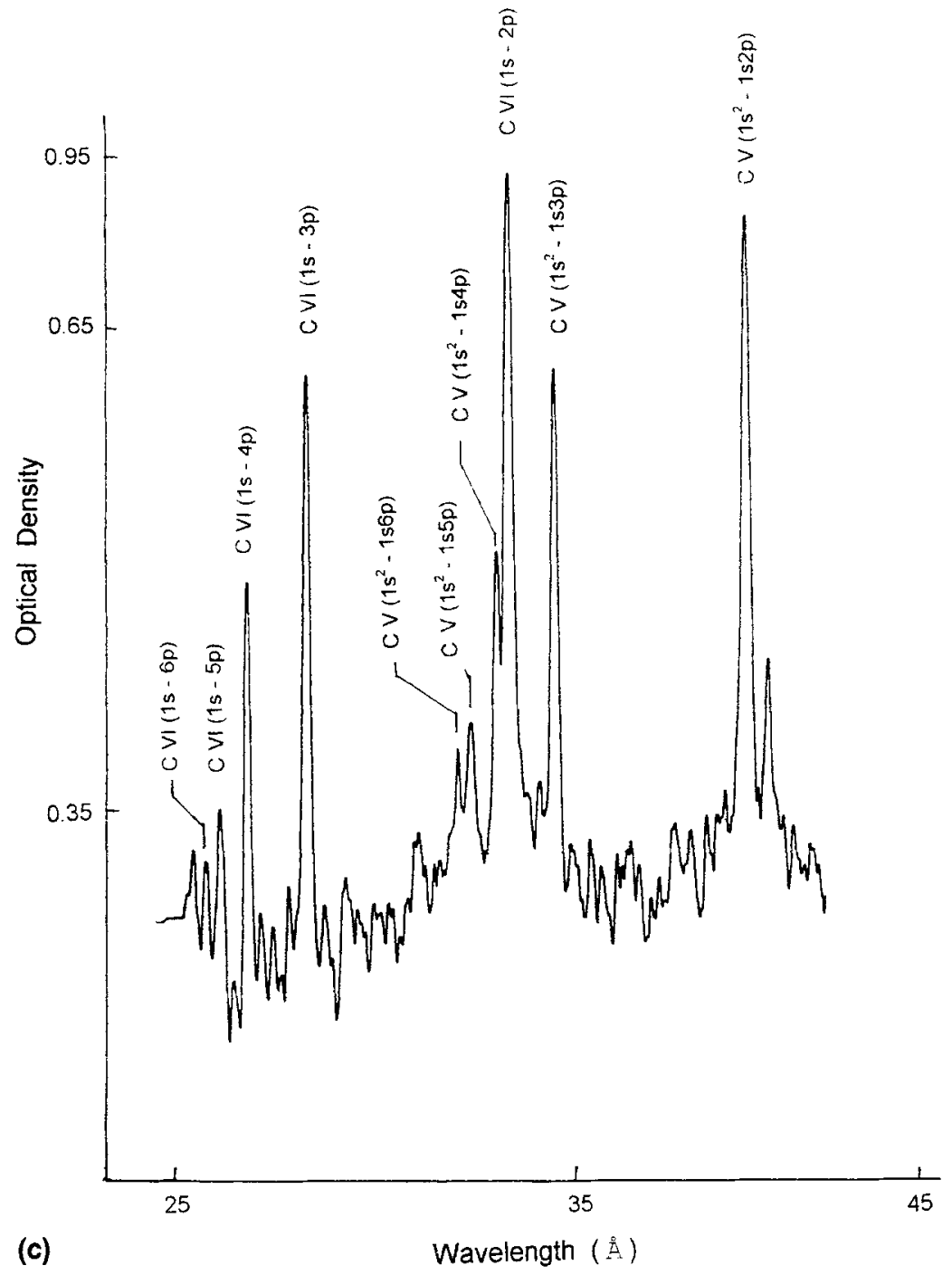

Figure 5. Spectrum of laser-produced carbon plasma at a laser intensity of $8 \times 10^{12} \mathrm{~W} / \mathrm{cm}^{2}$. (a) Spectrum recorded over full spectral range $(25-290 \AA)$. (b) Expanded view of the spectrum for $\lambda<44 \AA$. (c) Densitometric trace of the spectrum corresponding to figure $5 \mathrm{~b}$.

$(\mathrm{He} \beta)$ at $35.0 \AA, \mathrm{CV} 1 s^{2}{ }^{1} S_{0}-1 s 4 p^{1} P_{1}(\mathrm{He} \gamma)$ at $33.4 \AA$, $\mathrm{CV} 1 s^{2}{ }^{1} S_{0}-1 s 5 p{ }^{1} P_{1}(\mathrm{He} \delta)$ at $32.8 \AA$, $\mathrm{CV} 1 s^{2}{ }^{1} S_{0}-1 s 6 p^{1} P_{1}(\mathrm{He} \epsilon)$ at $32.4 \AA$, and for the H-like carbon ions CVI $1 s^{2} S_{1 / 2}-2 p^{2} P_{3 / 2}$ $(\mathrm{Ly} \alpha)$ at $33.7 \AA$, CVI $1 s^{2} S_{1 / 2}-3 p^{2} P_{3 / 2}(\mathrm{Ly} \beta)$ at $28.5 \AA$, CVI $1 s^{2} S_{1 / 2}-4 p^{2} P_{3 / 2}$ (Ly $\left.\gamma\right)$ at $27.0 \AA$, CVI $1 s^{2} S_{1 / 2}-5 p^{2} P_{3 / 2}(\mathrm{Ly} \delta)$ at $26.4 \AA$, CVI $1 s^{2} S_{1 / 2}-6 p^{2} P_{3 / 2}$ (Lye) at $26.0 \AA$.

An estimate of the spectral resolution can be obtained from the FWHM of a sharp line transition observed in the spectrum. For instance, at $\lambda=35.0 \AA$, the FWHM of the CV $1 s^{2}-1 s 2 p$ line were taken as an upper bound on the spectral resolution. Data on the $\gamma$ (slope of the optical density versus log (exposure) curve) of the film for the same conditions of processing were taken from Alexandrov et al (1991). Variation of spectral resolution with respect to the distance of film plane from the grating centre is shown in figure 6 . 


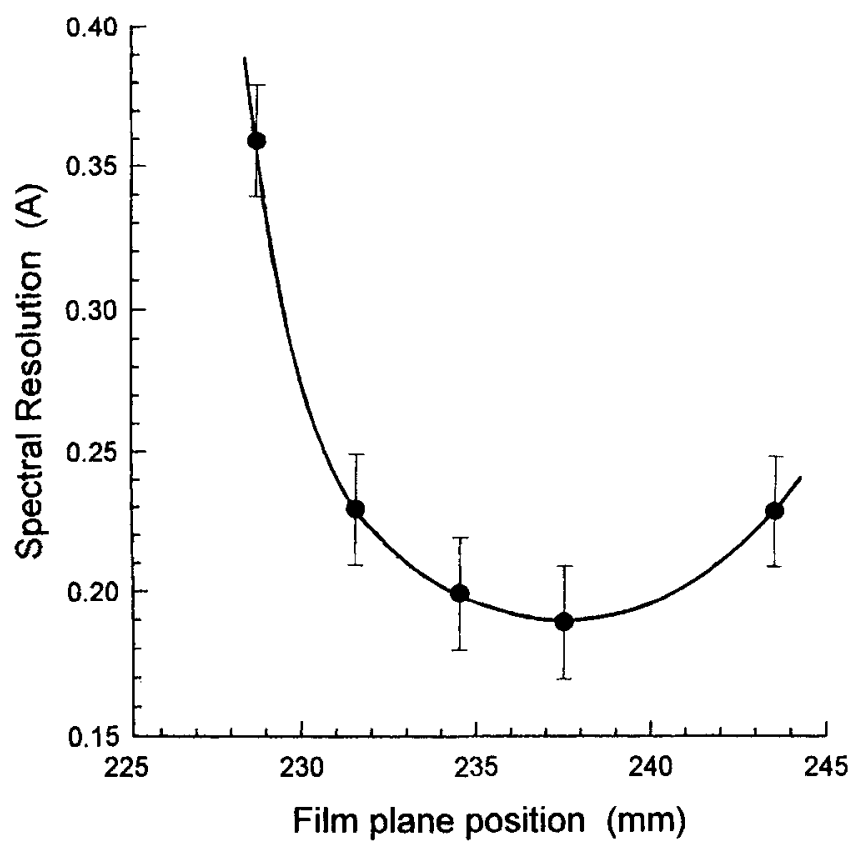

Figure 6. Variation of spectral resolution with film plane position.

It is noted that the best spectral resolution of $0.2 \AA$ (corresponding resolving power $\lambda / \Delta \lambda \approx 175$ ) is achieved at a distance of about $237 \mathrm{~mm}$ from the grating centre. This spectral resolution is also evident from figure $5 \mathrm{c}$ which shows that closely placed spectral lines $\mathrm{CV} 1 s^{2}{ }^{1} S_{0}-1 s 4 p{ }^{1} P_{1}$ at $\lambda=33.4 \AA$ and $\mathrm{CVI} 1 s^{2} S_{1 / 2}-2 p^{2} P_{3 / 2}$ at $\lambda=33.7 \AA$, are clearly resolved from each other. Further, the observed distance of focal plane from the grating centre of $237 \mathrm{~mm}$ is also close to the theoretical design value of $235 \mathrm{~mm}$. The small difference may be due to alignment tolerance on the angle of incidence of $87^{\circ}$ used in the setup. It may however be noted from figure 6 that the spectral resolution degrades only marginally to $0.22 \AA$ for a $\pm 5 \mathrm{~mm}$ change from the best focal position. This clearly shows the superiority of the flat-field focussing configuration over the Rowland geometry, where a much more precise matching of the detector surface to the Rowland circle is necessary.

The observed spectral resolution of $0.2 \AA$ may now be compared with the theoretically expected value from design parameters. Taking a plate factor of $3.7 \AA / \mathrm{mm}$ for $\lambda=35 \AA$ and a slit width of 50 micron (imaged with unit magnification on the detector plane), the spectral resolution is expected to be $0.185 \AA$. Thus the experimentally measured value of resolution matches well with the slit width limited theoretically calculated value of resolution. Next the spectral resolution was also checked in the higher wavelength region of the spectrograph operation. For CVI $2 s^{2} S_{1 / 2}-4 p^{2} P_{1 / 2,3 / 2}$ transition at $\lambda=134.9 \AA$, the FWHM of the line is experimentally estimated to be $\approx 0.5 \AA$ using an extrapolated value of $\gamma$ (Alexandrov et al 1991). The slit width limited value of resolution expected at this wavelength is $0.32 \AA$. It may be noted that the experimentally estimated value is only an upper bound on the true spectral resolution. The observed difference between the theoretically calculated and experimentally measured values may be attributed to a larger Doppler broadening at higher wavelengths. Nevertheless, the measured spectral resolution is good enough to carry out experiments involving population inversion and higher order harmonics in the XUV spectral region. 


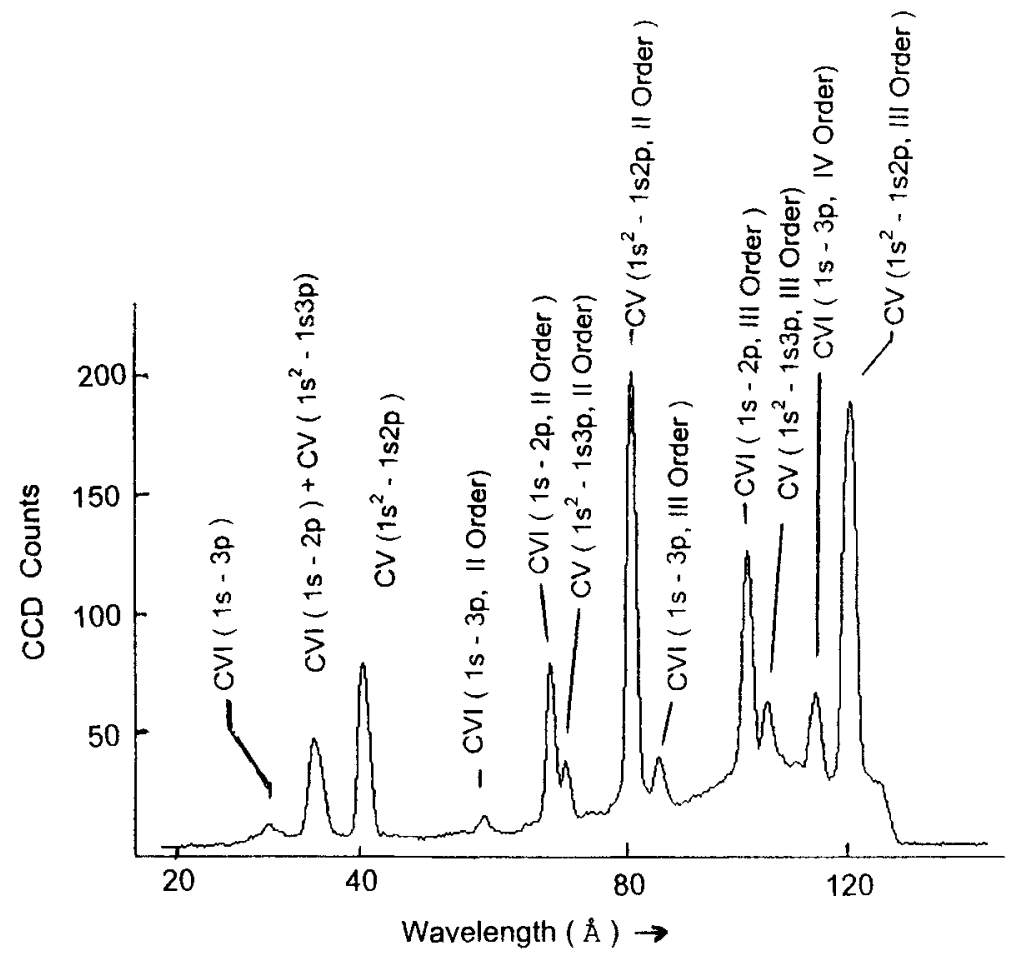

Figure 7. A typical spectrum of carbon plasma using MCP-CCD camera detection system.

Estimation of plasma temperature was carried out from intensity ratios of $\mathrm{He} \alpha$ and $\mathrm{He} \beta$ of carbon lines using the spectroscopic code RATION (Lee et al 1990). This code calculates ratios of various line intensities versus electron densities for different electron temperatures or line intensity ratio versus electron temperature for different values of electron densities. The contribution of the background continuum on which the lines are superimposed, is subtracted for obtaining the line intensities. The temperature was estimated to be $130 \pm 10 \mathrm{eV}$ and the density was $6 \times 10^{20} \mathrm{~cm}^{-3}$. This result is typically expected for the plasma produced at the laser intensity used in the experiment.

Although spectrograph performance is measured using X-ray photographic film as the detector, flat-field focusing allows coupling of real-time detectors like microchannel plateCCD cameras for on-line monitoring and analysis of the spectrum. Operation of the spectrograph in on-line mode was checked by using a 25 -millimetre diameter single-stage open type MCP and recording the spectra with a CCD camera frame-grabber system. A typical intensity versus wavelength scan of the carbon plasma spectrum using this system is shown in figure 7 . Intensity calibration and optimization of this detector performance is underway and will be reported separately.

\section{Conclusion}

A grazing incidence flat-field focussing grating spectrograph operating in the wavelength range of $25-290 \AA$ has been set up. Optimization of spectral resolution with respect to the 
detector plane position is studied, and the observed spectral resolution of $0.2 \AA$ is in agreement with that expected from design considerations. The spectrograph can record single shot spectra of laser-produced plasmas using either XUV-soft X-ray films or MCPCCD camera detection system. With its simple construction and observed spectral resolution, this spectrograph should be very useful for laser-plasma interaction studies, particularly in investigations of population inversion and high harmonic generation in the XUV spectral region.

\section{References}

Alexandrov Yu M et al 1991 Investigation of sensitometric characteristics of X-ray photoemulsions in the spectral range of 15-80 А. Nucl. Instrum. Methods Phys. Res. A308: 343-346

Chaker M, Bareau V, Kieffer J C, Pepin H, Johnston T W 1989 Calibrated soft X-ray spectroscopy using transmission grating, pinhole and film. Rev. Sci. Instrum. 60:3386-3390

Chaker M et al 1992 Laser plasma sources for proximity printing or projection X-ray lithography. $J$. Vac. Sci. Technol. B10: 3239-3242

Chakera J A, Kumbhare S R, Gupta P D 1998 Characterisation of X-ray contact microscopic imaging in keV spectral region using laser produced plasmas. J. X-ray Sci. Technol. 8: 1-9

Ditmire Tet al 1995 Amplification of XUV harmonic radiation in a gallium amplifier. Phys. Rev. A51: $4337-4340$

Eidmann K, Kishimoto T, Herrmann P, Mizui J, Pakula R, Sigel R, Witkowski S 1986 Absolute soft $\mathrm{X}$-ray measurements with a transmission grating spectrometer. Laser and Particle Beams 4: 521-526

Hogan W G 1995 Energy from inertial fusion. ST1/PUB/944, International Atomic Energy Agency (IAEA) Vienna

Huillier A H, Balcou P 1993 High order harmonic generation in rare gases with a 1 psec $1053 \mathrm{~nm}$ laser. Phys. Rev. Lett. 70: 774-777

Kita T, Harada T, Nakano N, Kuroda H 1983 Mechanically ruled aberration corrected concave gratings for a flat field grazing incidence spectrograph. Appl. Opt. 22: 512-513

Kiehn P, Willi O, Damerell A R, Key M H 1987 Novel time-resolved XUV spectrograph for X-ray laser research. Appl. Opt. 26: 425-426

Kelly R L, Palumbo L J 1973 Atomic and ionic emission lines below 2000 A. National Research Laboratory Report 7599

Lee R W, Strout R E, Cauble R C, Petway L B 1990 "RATION Spectroscopic Code", Lawrence Livermore National Laboratory, USA

Miyazaki K, Takada H 1995 High order harmonic generation in the tunneling regime. Phys. Rev. A52: 3007-3021

Matthews D L et al 1985 Demonstration of soft X-ray amplifier. Phys. Rev. Lett. 54: 110-113

Nagata Y, Midorikawa K, Kubodera S, Obara M, Tashiro H, Toyoda K 1993 Soft X-ray amplification of Lyman alpha transition by optical field induced ionisation. Phys. Rev. Lett. 71: 3774-3777

Nakano N, Kuroda H, Kita T, Harada T 1984 Development of a flat field grazing incidence spectrometer and its application in picosecond XUV spectroscopy. Appl. Opt. 23: 2386-2392

Stead A D, Cotton R A, Duckett J G, Goode J A, Page A M, Ford T W 1995 The use of soft X-rays to study the ultrastructure of living biological material. J. X-ray Sci. Technol. 5: 52-64

Sailaja S, Arora V, Kumbhare S R, Naik P A, Gupta P D, Fedin D A, Rupasov A A, Shikanov A S 1998a A simple XUV transmission grating spectrograph with sub $\AA$ resolution for laser plasma interaction studies. Meas. Sci. Technol. 9: 1462-1468

Sailaja S, Arora V, Kumbhare S R, Naik P A, Gupta P D 1998b Study of diffraction efficiency of a free-standing transmission grating in $\mathrm{keV}$ spectral region using laser produced plasmas. Opt. Laser Technol. 30: 407-410

Schwanda N, Eidmann K, Richardson M C 1993 Characterization of a flat field grazing incidence spectrometer. J. X-ray Sci. Technol. 4: 8-17 